\title{
STRIPPING OF A PLANETARY NEBULA FROM THE GLOBULAR CLUSTER M22
}

\author{
K.J. BORKOWSKI
}

Department of Astronomy, University of Maryland, College Park, MD 20742, USA J.P. HARRINGTON

Department of Astronomy, University of Maryland, College Park, MD 20742, USA and

\section{Z. TSVETANOV}

Department of Physics and Astronomy, The Johns Hopkins University, Baltimore, USA

High-spatial resolution imaging in the [O III] $\lambda 5007$ line of a planetary nebula (PN) in the globular cluster M22 reveals a strongly asymmetric (a half-moon shaped) nebular morphology. We confirm that this peculiar morphology is caused by the distortion of stellar ejecta by the ram pressure of the ambient interstellar medium (ISM) through which the cluster is moving with a high velocity of $200 \mathrm{~km} \mathrm{~s}^{-1}$. Bright emission knots visible in the leading (upstream) nebular section confirm theoretical expectations that the shell should have been fragmented by the Rayleigh-Taylor instability. Stripping of the PN from M22 by the ambient ISM is the first direct evidence for removal of gas from globular clusters. M22 is located at a favourable location for the ram-pressure stripping to be effective, only $400 \mathrm{pc}$ below the Galactic plane, well within a $2 \mathrm{kpc}$ thick layer of ionized gas enveloping the plane. Ionized (possibly neutral) gas in this layer, with a hydrogen density of $\sim 0.1 \mathrm{~cm}^{-3}$, is responsible for the observed interaction. 